\title{
Dynamic Route Discovery Using Modified Grasshopper Optimization Algorithm in Wireless Ad-Hoc Visible Light Communication Network
}

\author{
Sharmila Vadivel ${ }^{1}$, Srinivas Konda ${ }^{2}$, Kavitha Rani Balmuri ${ }^{3}$, Andrzej Stateczny ${ }^{4, *(1)}$ and B. D. Parameshachari ${ }^{5}$ (i) \\ 1 Department of Computer Science and Engineering, K. S. R. College of Engineering, \\ Tiruchengode 637215, India; sharmila@ksrce.ac.in \\ 2 Department of Computer Science and Engineering, CMR Technical Campus, Hyderabad 501401, India; \\ Srinivas.cse@cmrtc.ac.in \\ 3 Department of Information Technology, CMR Technical Campus, Hyderabad 501401, India; \\ Kavitharani.cse@cmrtc.ac.in \\ 4 Department of Geodesy, Gdańsk University of Technology, Gabriela Narutowicza 11-12, \\ 80-233 Gdańsk, Poland \\ 5 Department of Telecommunication Engineering, GSSS Institute of Engineering and Technology for Women, \\ Karnataka 570016, India; paramesh@gsss.edu.in \\ * Correspondence: andrzej.stateczny@pg.edu.pl
}

Citation: Vadivel, S.; Konda, S.; Balmuri, K.R.; Stateczny, A.;

Parameshachari, B.D. Dynamic Route Discovery Using Modified

Grasshopper Optimization Algorithm in Wireless Ad-Hoc Visible Light Communication Network. Electronics 2021, 10, 1176. https://doi.org/ 10.3390/electronics10101176

Academic Editor: Paul Mitchell

Received: 14 April 2021

Accepted: 12 May 2021

Published: 14 May 2021

Publisher's Note: MDPI stays neutral with regard to jurisdictional claims in published maps and institutional affiliations.

Copyright: (c) 2021 by the authors. Licensee MDPI, Basel, Switzerland. This article is an open access article distributed under the terms and conditions of the Creative Commons Attribution (CC BY) license (https:// creativecommons.org/licenses/by/ $4.0 /)$.

\begin{abstract}
In recent times, visible light communication is an emerging technology that supports high speed data communication for wireless communication systems. However, the performance of the visible light communication system is impaired by inter symbol interference, the time dispersive nature of the channel, and nonlinear features of the light emitting diode that significantly reduces the bit error rate performance. To address these problems, many environments offer a rich infrastructure of light sources for end-to-end communication. In this research paper, an effective routing protocol named the modified grasshopper optimization algorithm is proposed to reduce communication interruptions, and to provide alternative routes in the network without the need of previous topology knowledge. In this research paper, the proposed routing protocol is implemented and analyzed using the MATLAB environment. The experimental result showed that the proposed routing protocol adapts to dynamic changes in the communication networks, like obstacles and shadows. Hence, the proposed protocol achieved better performance in data transmission in terms of throughput, packet delivery ratio, end-to-end delay, and routing overhead. In addition, the performance is analyzed by varying the number of nodes like 50, 100, 250, and 500. From the experimental analysis, the proposed routing protocol achieved maximum of $16.69 \%$ and minimum of $2.20 \%$ improvement in packet delivery ratio, and minimized 0.80 milliseconds of end-to-end delay compared to the existing optimization algorithms.
\end{abstract}

Keywords: communication network; grasshopper optimization algorithm; optimal routing; visible light communication; wireless ad hoc network

\section{Introduction}

Due to the modern problems in wireless communication system, the Wireless Ad-hoc Visible Light Communication Network (WAVLCN) has become a popular technology among industrial and academic communities [1-3]. The Visible Light Communication (VLC) is applied in an extensive range of applications: underwater communication [4], localization networks [5], vehicular networks [6], and indoor communication networks [7]. The VLC system consists of two major elements, like receiver and transmitter, where a Light Emitting Diode (LED) is used as a transmitter that modulates the light intensity for transmitting the data [8]. On the other hand, the photo-sensors are utilized to capture light at the receiver side, and then convert light into data streams [9]. In the VLC network, the LEDs transmit light by 
using its field of view that reduces the angle of emission, so it works on the basis of sight line between transmitter and receiver [10]. However, the VLC system has several issues during data transmission, like shadowing and obstacles [11,12]. To address these problems, a new dynamic routing protocol is proposed in this research paper.

A dynamic routing protocol named the Modified Grasshopper Optimization Algorithm (MGOA) is proposed as a cross-layer routing protocol for WAVLCN. In this research, the proposed routing protocol is inspired from the mobile ad hoc network routing protocols [13]. On the basis of mobile ad hoc network routing protocols, the proposed MGOA routing protocol is created using VLC by adapting to network change [14,15]. In this research paper, the main contribution of MGOA is its integration with the Medium Access Control (MAC) protocol, named as Carrier Sense Multiple Access with Collision Avoidance (CSMA/CA), which is applied for routing maintenance. The CSMA/CA protocol makes visible light highly sensible to shadows, and obstacles. The performance analysis of the proposed model is done by using several performance metrics such as Packet Delivery Ratio (PDR), end-to-end delay, throughput, and routing overhead. From the simulation results, the proposed routing protocol almost reduced $0.3 \mathrm{~s}$ of end-to-end delay in data transmission and showed 3\% to 8\% improvement in PDR compared to the existing protocol.

This article is pre-arranged as follows. Some recent research works on the topic "VLC" are surveyed in Section 2. The proposed MGOA-WAVLCN is briefly explained in Section 3. The experimental evaluation of the proposed MGOA-WAVLCN is given in Section 4. The conclusion of the research work is represented in Section 5.

\section{Related Works}

L.M. Matheus et al. [16] developed a new Dynamic Routing Protocol (DYRP) in wireless Ad Hoc VLC network to diminish interruptions during communication, and to generate alternative routes in the VLC networks without the need of prior topology knowledge. Experimental result showed that the dynamic routing protocol significantly deals with the obstacles and shadows in the VLC networks by generating alterative paths between the source and destination nodes. The developed dynamic routing protocol needs to address the security processes for protecting the network and its nodes in order to ensure a correct route between the nodes. In addition, S. Lou et al. [17] developed a new two-step algorithm for reducing the optimization problem with low implementation complexity in the VLC networks. The numerical outcome showed that the developed algorithm achieved optimal solution compared to brute force technique in light of total transmission power. W.X. Hong et al. [18] presented a new channel access model-CSK hopping pattern model for VLC. In this literature, the transmitter utilizes the CSK hopping pattern model for loading the data into Light Emitting Diodes (LEDs). Next, the network access point of the LEDs was established for illumination coverage area, where it uses dissimilar color patterns for distinguishing the VLC networks. The developed model establishes a communication access network process with the access point, while a receiver detects a specific color pattern in the illumination coverage area. The developed CSK hopping pattern model effectively minimizes the delay in the terminal access network and interference between the adjacent access points. However, the developed CSK hopping pattern model was used only in special drive dimmer circuits, and also it was hard to balance the communication and lighting.

G. Sun et al. [19] developed an improved cuckoo search algorithm on the basis of dimension cells and chaos theory to solve the uneven distribution of received optical power. In this literature, the chaos process optimizes the random distribution of the initial solution for reducing the interference. The experimental outcome showed that the developed improved cuckoo search algorithm obtained better convergence rate and accuracy compared to other metaheuristics optimization algorithms in VLC. Still, the developed algorithm needs to solve the problems concerning coverage and network access in smart homes. C. Biton and S. Arnon [20] developed a heuristic allocation technique for reducing the system sub-carrier transmission power, while maintaining the required error bit rate and data 
rate. The numerical analysis showed that the developed technique effectively minimizes the clipping noise, transmitter power consumption, and multi-user interference at the receiver end. The numerical results showed that the developed technique reduces more than $10 \%$ of transmitter power consumption compared to the existing techniques. As a future enhancement, the developed technique needs to concentrate on the energy efficiency of the communication system. F. Li et al. [21] analyzed the energy saving ability of the dimming VLC system by using the Sub Carrier-4 Pulse Position Modulation (SC-4 PPM) technique. In this literature study, different optimization algorithms were applied for maximizing the energy efficiency of VLC network. The trade-off between Signal-to-Noise Ratio (SNR) and energy efficiency were uniform to obtain efficient performance. The potential of LED parameters to enhance VLC energy efficiency has not been intensively analyzed in this study.

J.P. Ding and Y.F. Ji [22] introduced an evolutionary algorithm for optimizing the SNR distribution in an indoor VLC system. The numerical results showed that the developed evolutionary algorithm almost reduced $25 \%$ of SNR deviations, which was better compared to other algorithms. By using the general concepts, the evolutionary algorithm finds the set of factors that corresponds to different system configurations, where it cannot be applicable in real-world applications. H. Liu et al. [23] implemented a Gene Density Genetic Algorithm (GDGA) for identifying the optimized lighting layout in the VLC network. The gene mutation and crossover techniques in GDGA uniforms the received power on the communication plane. Based on the fitness function of GDGA, a weighted difference function between the individuals was designed to reserve the population that have LED layout genetic information. The optimized layout has better power uniformity compared to square layout and circular layout. However, the convergence rate of GDGA was a bit lower compared to the evolutionary algorithm, which needs to be concentrated on as a future extension.

H. Liu et al. [24] developed an Improved Genetic Simulated Annealing Algorithm (IGSAA) to improve the fairness and minimize the power fluctuations in the VLC system by finding the optimal power factors without changing the LED location and layouts. In this literature, dynamic mutation probability and two-point crossover operation were developed based on the information of optimal chromosome and evolutional generation to speed up the search coverage. In IGSAA, a metropolis criterion was utilized for maintaining the population diversity. Numerical analysis showed that the developed IGSAA significantly improves the coverage uniformity compared to the prior algorithm in the indoor VLC network. Additionally, it was difficult to identify the global optimal solution of the objective problem in IGSAA to improve coverage uniformity. W. Jiaan et al. [25] implemented an Improved Artificial Fish Swarm Algorithm (IAFSA) for identifying optimal LED layout for better communication. The artificial fish indicates LED position in IAFSA that contains a pair of ordinate and abscissa. The order technique in IAFSA effectively increases the searching efficiency and the ability of global search, which ensures the convergence. The IAFSA optimized layout has better power uniformity augments related to circular layout, rectangular layout, and square layout. A uniform power distribution over the communication plane is an issue in the IAFSA, which may increase the Mean Square Error (MSE) of the receiving power. H. Yuksel and Ö. Altunay [26] enabled a hostto-host internet protocol and transmission control protocol connection over serial ports by using VLC. In this research, the data was transmitted from the serial ports to the virtual tunneling devices, so that the processor receives the data without utilizing the Ethernet connections. A ping tool was used in the study for checking the network connection status between two computers. The ping tool checks whether it is possible to reach one computer from another computer. The performance measures power efficiency and data rate confirms that the developed protocol connection was effective in VLC systems. In addition, A. Faisal et al. [27] utilized a combination of methods-threshold combining, selection combining, and maximal ratio combining-for reducing the receiver outage probability. The simulation outcomes confirmed that the developed method outperforms the existing 
methods in terms of outage probability and signal-to-noise ratio. However, the VLC suffers from line of sight constraints and severe short communication ranges. In order to address the above stated issues and to improve data transmission efficiency in WAVLCN, a new MGOA is proposed in this research article.

\section{Methodology}

In general, the VLC system includes components to receive-cameras and photo diode-and a component to transmit-LED [28]. In the VLC system, the data are modulated by utilizing the modulator and transmitted to the LEDs, which are responsible to transmit the light $[16,29,30]$. Here, the modulation of the data is carried out by varying the light intensity according to the information exists in the message signal. Similarly, the photosensors are responsible to capture the light directly at the receiver end and then convert the light into data stream. Moreover, the demodulator used in the receiver end is used to demodulate the data. The overview of the VLC system is graphically depicted in Figure 1.

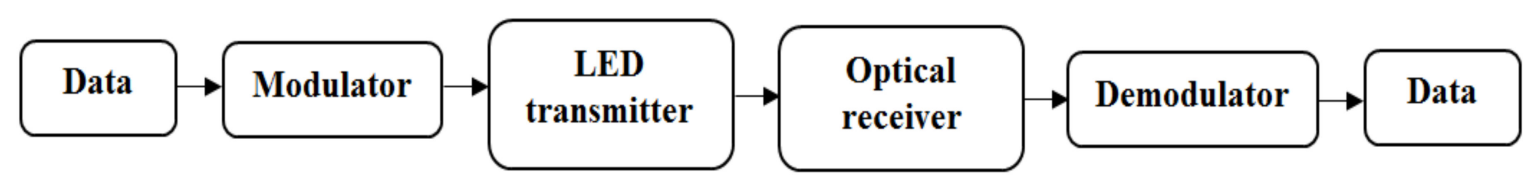

Figure 1. Overview of the VLC system.

The VLC networks have similar properties like mobile ad hoc networks, particularly while considering limited mobility, physical layer constraints, and bandwidth [31]. In this research paper, the drawbacks in the VLC scenarios are addressed using the reactive protocols in ad hoc networks. The main objective of the reactive protocols is to adapt the changes in the VLC networks by using its reactive characteristics [32,33].

\subsection{System Model}

The MGOA-WAVLCN system model is comprised of sensor nodes and several lighting sources to transmit and receive the data packets. The lighting infrastructure and the sensor nodes in the MGOA-WAVLCN model are equipped with transmitter and receiver for data transmission. In this scenario, the VLC transmitter and receiver were connected using a free space optical communication channel. In VLC, the light emitted from the transmitter is comprised of data packets, which are transmitted by using wireless medium. The channel model of MGOA-WAVLCN is mathematically indicated in Equation (1).

$$
M G O A-W A V L C N=\left\{\begin{array}{cc}
\frac{(B+1) M}{2 \pi d i s t^{2}} \cos ^{B}(\varphi) O(\psi) q(\psi) \cos (\psi) & 0 \leq \psi \leq \psi_{l} \\
0 & \psi>\psi_{l}
\end{array}\right\}
$$

where $q(\psi)$ indicates concentrator gain, $\psi_{l}$ represents receivers field of view, $\psi$ represents angle of impedance, $\varphi$ indicates angle of irradiance, $O(\psi)$ indicates optical band pass filter, dist denotes distance between one node to another node, $M$ represents detector area, and $B$ denotes order of the Lambertian emission. The workflow of MGOA-WAVLCN is represented in Figure 2. 


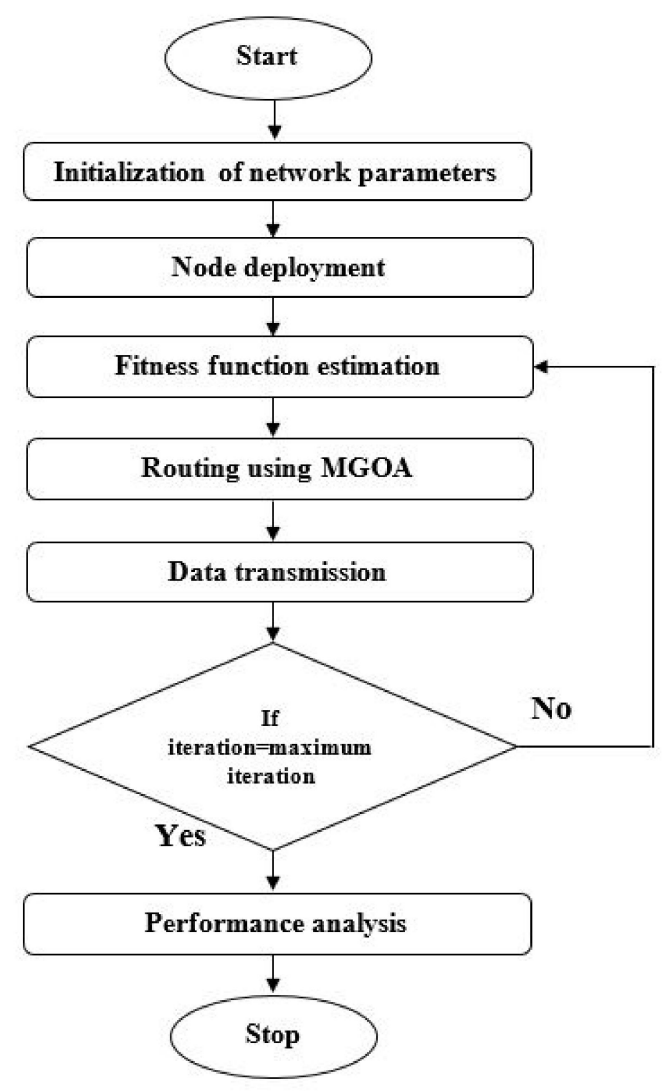

Figure 2. Workflow of MGOA-WAVLCN.

\subsection{Overview of $M G O A$}

The GOA is a modern optimization algorithm, which is used to solve the optimization issues like unconstrained and constrained issues. The GOA is a nature-based algorithm which mimics the swarming features of grasshopper [34]. The grasshopper location in the swarm exhibits the possible solution to the optimization issues. The location of $i^{\text {th }}$ grasshopper is represented as $X i$, which is mathematically determined in Equation (2).

$$
X i=S i+G i+A i
$$

where $A i$ indicates wind advection, Gi states gravitational force experienced by $i^{\text {th }}$ grasshopper, and $\mathrm{Si}$ states social interaction, where these three components replicates the grasshopper's motion. Among the three components, $S i$ is the important component, which is generated by the grasshoppers themselves and is mathematically represented in Equation (3).

$$
S i=\sum_{j=1, j \neq i}^{N} s\left(d_{i j}\right) \grave{d}_{i j}
$$

where $d_{i j}=|x j-x i|$, and $\grave{d}_{i j}=\frac{x j-x i}{d_{i j}}$ represents a unit vector. The function $s$ represents strength of social forces, which is mathematically depicted in Equation (4).

$$
s(r)=\frac{f e-r}{l-e-r}
$$

where $l$ states attractive length scale, and $f$ denotes intensity of attraction. In GOA, the separation between two grasshoppers is accomplished using repulsive forces that ranges between $(0,2.079)$. The attractive and offensive forces between two grasshoppers are equilibrium, while the separation between two grasshoppers is 2.079 units. In addition, 
the trajectory, repulsive forces, and attractive forces of the grasshoppers influence the swarming capacity [35].

The grasshopper's separation is classified into three regions, such as repulsion region, attraction region, and comfort zone, using function $s$, which is almost equal to zero. Next, the separation between the grasshoppers is mapped and the interval between them is set $(1,4)$. In addition, the component Gi mentioned in Equation (2) is mathematically defined in Equation (5).

$$
G i=-g e g
$$

where $e g$ denotes unity vector which directed towards the globe center, and $g$ states gravitational constant. Correspondingly, the component $A i$ mentioned in Equation (2) is mathematically defined in Equation (6).

$$
A i=\text { uew }
$$

where $e w$ indicates unity vectors, which uses the similar direction of wind, and $u$ states constant drift. The motion of nymph grasshoppers is highly correlated with the direction of wind blows and by substituting the components $G i, S i$, and Ai, Equation (7) is obtained.

$$
X i=j=1, j i N s\left(d_{i j}\right)\left(d_{i j}\right)-g e g+\text { uew }
$$

where $N$ indicates number of grasshoppers and $s(r)=\frac{f e-r}{l-e-r}$. The nymph grasshopper's location is avoided by reducing the threshold value while it reaches the ground. Equation (7) cannot be employed in the optimization algorithms and swarm simulation because it hinders the optimization algorithms from exploiting and exploring the search space near the solution, where this model is only used in the free space. In a swarm, the interaction between the grasshoppers is modeled by utilizing Equation (6). Generally, the optimization algorithm should carry out exploitation and exploration constructively for determining the accurate approximation of global optimum. Hence, the updated Equation (7) is indicated in Equation (8).

$$
X i d=c j=1, j i N c u b d-I b d 2 s(|x j d-x i d|) x j-x i d_{i j}+T d
$$

where $u b d$ indicates upper bound, Ibd states lower bound, Td denotes the value of $d^{\text {th }}$ dimension in targets, and $c$ represents decreasing coefficient, which is utilized for reducing the comfort region, repulsion region, and attraction region. In addition to this, $s$ is equivalent of $S$ component, as stated in Equation (2). The inner $c$ decreases the attractive or repulsive forces between two grasshoppers, and the outer $c$ decreases the search coverage by increasing the iteration. The $c u b d-I b d 2$ cause's linear reduction of the exploitation, and exploration space of the grasshoppers, and $T d$ represents habitual nature of the grasshoppers. Hence, the coefficient $c$ is directly proportional to the number of iterations, which is mathematically depicted in Equation (9).

$$
C=c \max -I c \max -\operatorname{cmin} \times \text { Maxitr }
$$

where $I$ denotes current iteration, cmin represents minimum value, cmax states maximum value, and Maxitr denotes maximum number of iteration. In MGOA, initially set the parametric values such as $X(t)$, Maxitr, cmin, and cmax, and then the $c$ value is updated based on the normalization relationship between the grasshoppers. The random forest classifier is developed after the creation of the optimal solutions. The optimal set of the nodes is minimized and the tree root is selected based on the condition of leaf node. The optimal random forest is applied for predicting the optimal node and the most voted solution is considered as the final solution. The parameter setting of MGOA is given in Table 1. 
Table 1. Parameter setting of MGOA.

\begin{tabular}{ccc}
\hline Parameters & Description & Value \\
\hline$D$ & Population size & 22 \\
$L b$ & Lower bound value & 0 \\
$U b$ & Upper bound value & 11 \\
$C \min$ & Minimum value of $c$ & 0.00002 \\
$C \max$ & Maximum value of $c$ & 1 \\
$W f$ & Control number of nodes & 6 \\
$M$ & selected & 20 \\
\hline
\end{tabular}

The pseudocode of MGOA is represented in the following Algorithm 1.

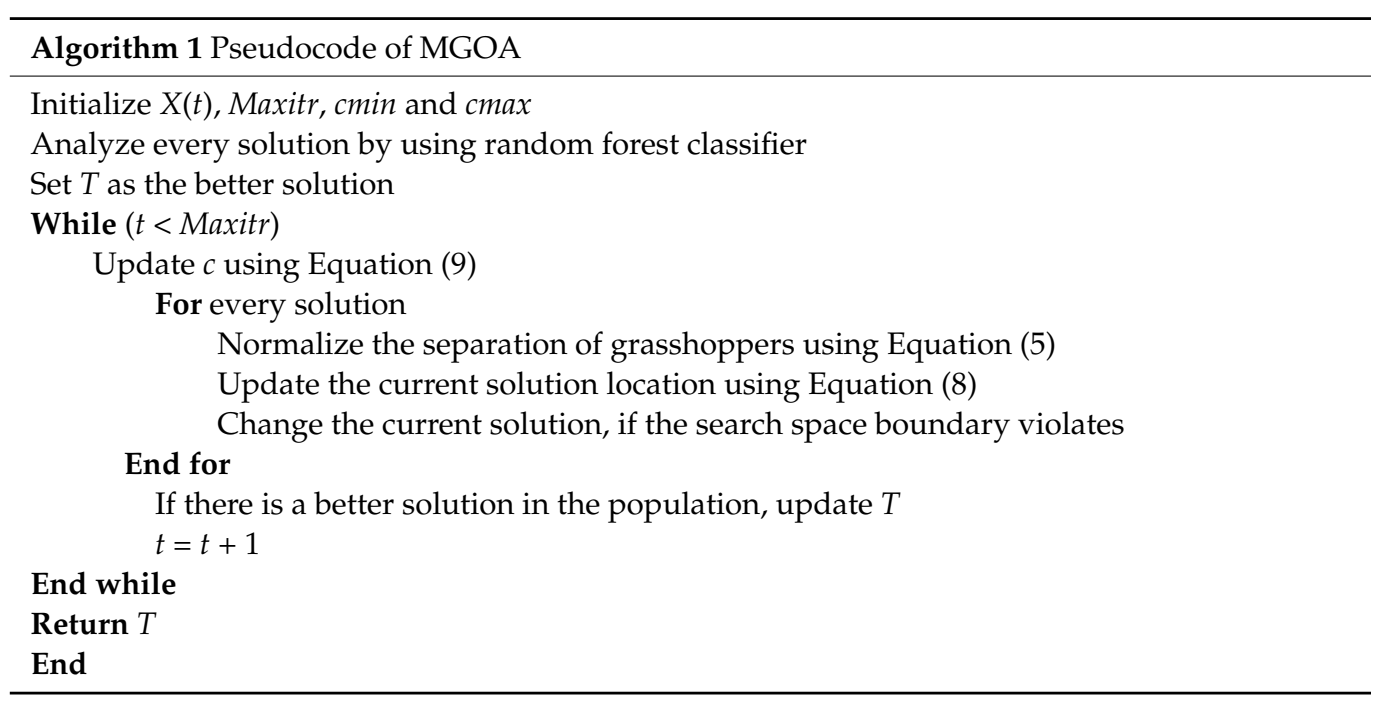

\subsection{Route Discovery Using MGOA}

The optimal route generation between the source and destination node using MGOA is explained in this section.

\subsubsection{Grasshopper Representation}

Each grasshopper in the MGOA is denoted the possible path from the source node to the destination node. Here, the grasshopper's dimension is identical to the amount of nodes in the possible paths.

\subsubsection{Grasshopper Initialization}

In the initialization phase, a grasshopper's dimension is equal to the number of nodes in the data transmission path. Consider the $i$ th grasshopper for the MGOA is $X i=\left(X i_{1}, X i_{1}, . ., X i_{m}\right)$, where $m$ is equal to the number of nodes in the respective path. Moreover, each grasshopper position $X i_{j}, 1 \leq j \leq n$ is represented the node towards the destination node. Here, $n$ defines the number of nodes in the WAVLCN.

\subsubsection{Derivation of Fitness Function}

In this research, fitness function is formulated using routing metric, number of hops, and intra cluster distance between the nodes for selecting the dynamic routing in WAVLCN. A new routing metric is used to find how suitable a communication link to route information between the nodes. The routing metric considers communication link quality on the basis of received optical power $p_{r}$ [36], which is mathematically defined in Equation (10). 
Here, the multi-objective function is converted into single objective using the weighted values $\gamma_{1}, \gamma_{2}$, and $\gamma_{3}$, as shown in the Equation (10).

$$
p_{r}=\gamma_{1} p_{t} H(0)+\gamma_{2} N H+\gamma_{3} d i s t
$$

where $p_{t}$ denotes transmit optical power of nodes, $H(0)$ represents channel gain, $\mathrm{NH}$ denotes the number of hops for each node, and dist denotes the distance between the nodes. The weighted values such as $\gamma_{1}, \gamma_{2}$, and $\gamma_{3}$ are equal to the $0.5,0.3$, and 0.2 (i.e., $\sum_{i=1}^{3} \gamma_{i}=1$ ). Based on fitness function mentioned in Equation (10), an optimal data transmission path is selected in each iteration of the MGOA. Next, the determined best path (i.e., Target position $T d$ ) is used to update the positions of the grasshopper for the remaining iterations, as shown in the Equation (8). In order to perform effective data transmission, the nodes require a smaller number of hops. The nodes with a smaller number of hops is a more preferable choice for data transmission [37]. In addition, the third objective function is inter-cluster distance between the nodes. The nodes consume less energy to process the data if the transmission distance is at a minimum. In this scenario, the Euclidean distance measure is used to find the distance between the nodes. The experimental analysis of the proposed MGOA-WAVLCN is briefly explained in Section 4.

\section{Simulation Results}

In this section, the experimental evaluation of the proposed MGOA-WAVLCN was simulated by utilizing the MATLAB (2018a) software tool with the system requirements: 8GB RAM, Intel core i7 processor, windows 10 operating system, and 4TB hard disk. In this scenario, the WAVLCN is used as a communication channel to support data transmission between the nodes, and MGOA is applied as a routing protocol to find the dynamic routing between source and destination nodes. The number of nodes deployed in the network area are varied as 50,100,250, and 500, where the range of network area is $300 \times 300 \mathrm{~m}^{2}$ with the transmission range of $15 \mathrm{~m}$, and the maximum transmission distance is $20 \mathrm{~m}$ in the real-time scenarios. The parameter specification of MGOA-WAVLCN is stated in Table 2. The performance of the proposed MGOA-WAVLCN is investigated by means of throughput, PDR, routing overhead, and end-to-end delay. Moreover, the performances of the MGOA-WAVLCN is compared with the DYRP-VLC [16]. The description about undertaken performance metrics is given below,

Table 2. Parameter specification of MGOA-WAVLCN.

\begin{tabular}{cc}
\hline Parameter & Value \\
\hline Area & $300 \times 300 \mathrm{~m}^{2}$ \\
Number of nodes & $50,100,250$, and 500 \\
Transmission range & $15 \mathrm{~m}$ \\
Communication channel & WAVLCN \\
Routing protocol & MGOA \\
Packet size & $4000 \mathrm{bits}$ \\
MAC protocol & CSMA/CA \\
Maximum transmission distance & $20 \mathrm{~m}$ \\
\hline
\end{tabular}

The PDR is determined as the ratio of data packets transmitted successfully to the destination nodes and the total data packets generated for those destination nodes. In addition, PDR effectively determines the packet loss rate that limits the network throughput. The high PDR represents better routing protocol performance and the mathematical expression of PDR is denoted in Equation (11).

$$
P D R=\frac{\text { Received data packets }}{\text { Sent data packets }} \times 100
$$


Throughput is determined as the ratio of the total number of data packets successfully reached at the receiver and the time consumed by the receiver to collect the data. In addition, throughput is expressed either by bites per second (bit/sec) or bytes per second (byte/sec). The factors that affect the throughput are limited energy, limited bandwidth, high topology changes in the network, and unreliable communication between the nodes. The efficient network has a higher throughput, which is computed by using Equations (12) and (13).

$$
\begin{gathered}
\text { Throughput }=\frac{\text { File size }}{\text { Transmission time (bps) }} \\
\text { Transmission time }(\text { bps })=\frac{\text { File size }}{\text { Bandwidth (seconds) }}
\end{gathered}
$$

The routing over-head is determined as the ratio of the data packets successfully received at the receiver end and the number of control packets. The routing overhead is mathematically denoted in Equation (14). In addition to this, end-to-end delay is defined as the total time needed to transfer the data packets from the source to the destination node, which is mathematically indicated in Equation (15).

$$
\begin{aligned}
& \text { Routing overhead }=\frac{\text { Amount of data packets }}{\text { Amount of data packets success fully received at the receiver }} \\
& \text { End to end delay }=\frac{\text { Time needed to transfer the data packets }}{\text { Amount of data packets success fully received at the receiver }}
\end{aligned}
$$

\subsection{Quantitative Analysis}

In this section, the performance of the proposed MGOA-WAVLCN is analyzed in terms of throughput, PDR, routing overhead, and end-to-end delay. In addition, the performance is analyzed with different optimization algorithms; Particle Swarm Optimization (PSO) algorithm, Grey Wolf Optimization (GWO) algorithm, FSA, GOA, and MGOA on different numbers of nodes such as 50,100, 250, and 500. By investigating Table 3, the proposed routing protocol, MGOA, obtained better throughput compared to other optimization algorithms. As seen in Table 3, the routing protocol MGOA obtained 594, 589, 553, and 544 bits per seconds on 50,100, 250, and 500 nodes, respectively, which are better than other comparative algorithms. Additionally, the graphical analysis of MGOA-WAVLCN in terms of throughput is represented in Figure 3.

Table 3. Performance analysis of MGOA-WAVLCN in terms of throughput.

\begin{tabular}{ccccc}
\hline \multicolumn{5}{c}{ Throughput (bit/s) } \\
\hline \multirow{2}{*}{ Optimization Algorithm } & \multicolumn{4}{c}{ Number of Nodes } \\
& $\mathbf{5 0}$ & $\mathbf{1 0 0}$ & $\mathbf{2 5 0}$ & $\mathbf{5 0 0}$ \\
\hline PSO & 531 & 520 & 499 & 480 \\
GWO & 564 & 532 & 521 & 502 \\
FSA & 572 & 555 & 539 & 528 \\
GOA & 588 & 573 & 540 & 531 \\
MGOA & 594 & 589 & 553 & 544 \\
\hline
\end{tabular}

Correspondingly, in Table 4, the performance of the proposed MGOA-WAVLCN is analyzed by means of PDR. By inspecting Table 4, the proposed routing protocol MGOA achieved PDR of $93.28 \%, 90.12 \%, 88.90 \%$, and $84.10 \%$ on $50,100,250$, and 500 nodes, respectively. Compared to the existing optimization algorithms, the proposed routing protocol MGOA showed a maximum of $16.69 \%$ and minimum of $2.2 \%$ improvement in PDR. In this research study, the proposed routing protocol MGOA considered appropriate fitness function, which effectively avoids the packet loss during route generation. Graphical analysis of MGOA-WAVLCN in terms of PDR is indicated in Figure 4. 


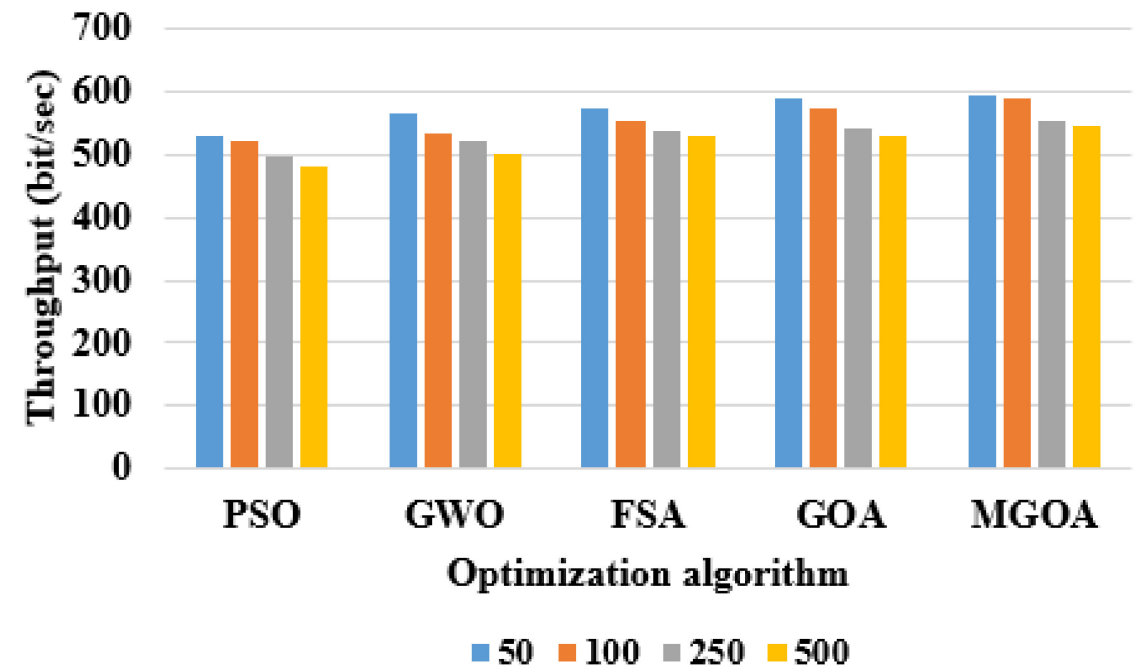

Figure 3. Graphical analysis of MGOA-WAVLCN in terms of throughput.

Table 4. Performance analysis of MGOA-WAVLCN in terms of PDR.

\begin{tabular}{ccccc}
\hline & \multicolumn{4}{c}{ PDR (\%) } \\
\hline \multirow{2}{*}{ Optimization Algorithm } & \multicolumn{4}{c}{ Number of Nodes } \\
& $\mathbf{5 0}$ & $\mathbf{1 0 0}$ & $\mathbf{2 5 0}$ & $\mathbf{5 0 0}$ \\
\hline PSO & 78 & 74.89 & 72.21 & 69 \\
GWO & 80 & 76.90 & 75 & 72 \\
FSA & 85 & 83.34 & 79 & 72.78 \\
GOA & 88 & 87.92 & 81 & 79.98 \\
MGOA & 93.28 & 90.12 & 88.90 & 84.10 \\
\hline
\end{tabular}

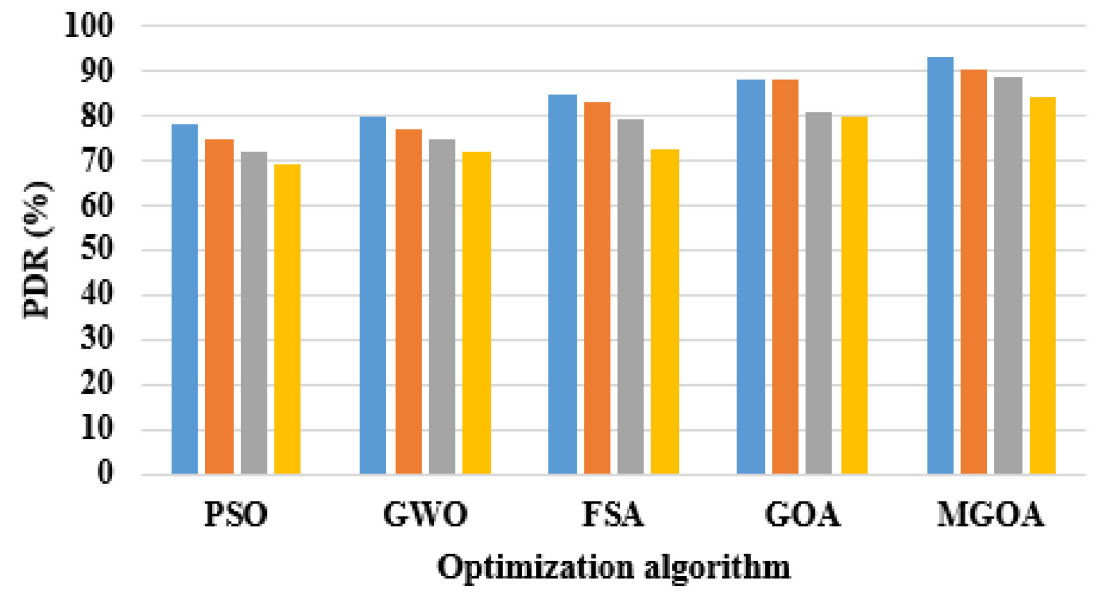

$\square 50 \square 100 \square 250 \square 500$

Figure 4. Graphical analysis of MGOA-WAVLCN in terms of PDR.

The performance analysis of MGOA-WAVLCN in terms of routing overhead is depicted in Table 5. By inspecting Table 5, the proposed routing protocol MGOA obtained $262,269,278$, and 283 routing overhead on 50, 100, 250, and 500 nodes, respectively, which are effective compared to other routing protocols such as PSO, GWO, FSA, and GOA. The overall experimental outcome showed that the relation between the average overhead and the route maintenance parameter are straightforward. Therefore, many route discovery attempts were done to find dynamic routing between source and destination nodes. The 
graphical analysis of MGOA-WAVLCN in terms of routing overhead is represented in Figure 5.

Table 5. Performance analysis of MGOA-WAVLCN in terms of routing overhead.

\begin{tabular}{ccccc}
\hline \multicolumn{5}{c}{ Routing Overhead } \\
\hline \multirow{2}{*}{ Optimization Algorithm } & \multicolumn{4}{c}{ Number of Nodes } \\
& $\mathbf{5 0}$ & $\mathbf{1 0 0}$ & $\mathbf{2 5 0}$ & $\mathbf{5 0 0}$ \\
\hline PSO & 349 & 357 & 370 & 398 \\
GWO & 316 & 322 & 324 & 333 \\
FSA & 298 & 312 & 312 & 320 \\
GOA & 280 & 288 & 298 & 301 \\
MGOA & 262 & 269 & 278 & 283 \\
\hline
\end{tabular}

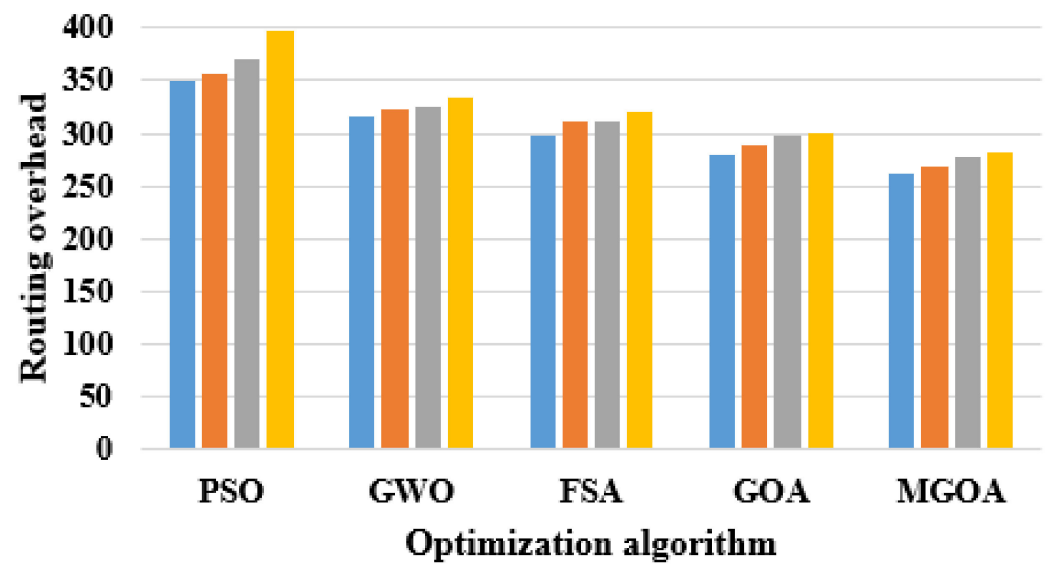

$$
\square 50 \square 100 \square 250 \square 500
$$

Figure 5. Graphical analysis of MGOA-WAVLCN in terms of routing overhead.

Similarly, the performance analysis of MGOA-WAVLCN in terms of end-to-end delay is stated in Table 6. By inspecting Table 6, the proposed routing protocol MGOA consumed only a less time for transmitting the data packets from source to the destination nodes. The proposed routing protocol MGOA consumed 2.09, 2.12, 2.19, and 2.31 milliseconds on 50, 100,250 , and 500 nodes, respectively, which are better compared to other optimization algorithms. Numerical analysis showed that the routing protocol MGOA approximately minimized 0.80 of a millisecond in relation to other routing protocols. The graphical analysis of MGOA-WAVLCN in terms of end-to-end delay is indicated in Figure 6.

Table 6. Performance analysis of MGOA-WAVLCN in terms of end-to-end delay.

\begin{tabular}{ccccc}
\hline \multicolumn{5}{c}{ End-To-End Delay (Milliseconds) } \\
\hline \multirow{2}{*}{ Optimization Algorithm } & \multicolumn{5}{c}{ Number of Nodes } \\
& $\mathbf{5 0}$ & $\mathbf{1 0 0}$ & $\mathbf{2 5 0}$ & $\mathbf{5 0 0}$ \\
\hline PSO & 2.89 & 2.97 & 3.02 & 3.18 \\
GWO & 2.81 & 2.86 & 2.99 & 3.09 \\
FSA & 2.65 & 2.76 & 2.88 & 2.92 \\
GOA & 2.44 & 2.59 & 2.70 & 2.86 \\
MGOA & 2.09 & 2.12 & 2.19 & 2.31 \\
\hline
\end{tabular}




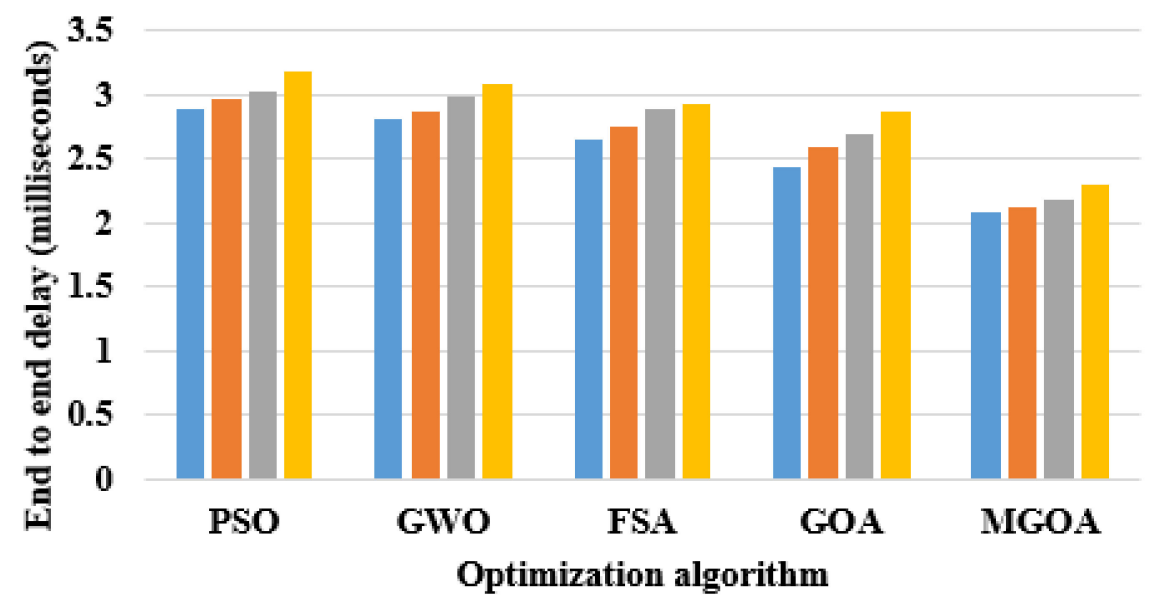

$\square 50 \quad \square 00 \square 250 \square 500$

Figure 6. Graphical analysis of MGOA-WAVLCN in terms of end-to-end delay.

\subsection{Comparative Analysis}

To justify the effectiveness of the proposed MGOA-WAVLCN, it was compared with one existing method, namely DYRP-VLC [16]. For analyzing the performances, the DYRPVLC [16] was also implemented and simulated for the same specifications mentioned in the Table 2.

Table 7 shows the performance analysis for the comparison between the DYRPVLC [16] with MGOA-WAVLCN. Here, the comparison is taken for varying nodes (i.e., $50,100,250$, and 500). From the comparison, it is concluded that the MGOA-WAVLCN outperforms the DYRP-VLC [16], because the DYRP-VLC [16] was considered only the hop count during the route generation. Moreover, the routing overhead of the DYRP-VLC [16] was high, because it continuously transmitted the request messages for a certain number of times to identify the transmission path. However, the MGOA-WAVLCN considers important parameters in the fitness function such as transmitting optical power of nodes, channel gain, number of hops, and distance. Therefore, the MGOA with an appropriate fitness parameter is used to select an optimal node during the route generation. In the routing path generation, the failure node is avoided by considering the optical power of the nodes in the MGOA which was used to improve the PDR.

Table 7. Comparison of the MGOA-WAVLCN and DYRP-VLC.

\begin{tabular}{|c|c|c|c|c|c|c|c|c|}
\hline \multirow{2}{*}{ Number of Nodes } & \multicolumn{2}{|c|}{ Throughput (bit/s) } & \multicolumn{2}{|c|}{ PDR (\%) } & \multicolumn{2}{|c|}{ Routing Overhead } & \multicolumn{2}{|c|}{$\begin{array}{l}\text { End-To-End Delay } \\
\text { (Milliseconds) }\end{array}$} \\
\hline & $\begin{array}{c}\text { DYRP-VLC } \\
\text { [16] }\end{array}$ & $\begin{array}{l}\text { MGOA- } \\
\text { WAVLCN }\end{array}$ & $\begin{array}{c}\text { DYRP-VLC } \\
\text { [16] }\end{array}$ & $\begin{array}{l}\text { MGOA- } \\
\text { WAVLCN }\end{array}$ & $\begin{array}{c}\text { DYRP-VLC } \\
\text { [16] }\end{array}$ & $\begin{array}{l}\text { MGOA- } \\
\text { WAVLCN }\end{array}$ & $\begin{array}{c}\text { DYRP-VLC } \\
\text { [16] }\end{array}$ & $\begin{array}{l}\text { MGOA- } \\
\text { WAVLCN }\end{array}$ \\
\hline 50 & 546 & 594 & 90.74 & 93.28 & 279 & 262 & 2.39 & 2.09 \\
\hline 100 & 507 & 589 & 86.33 & 90.12 & 283 & 269 & 2.46 & 2.12 \\
\hline 250 & 486 & 553 & 80.74 & 88.90 & 296 & 278 & 2.52 & 2.19 \\
\hline 500 & 439 & 544 & 78.11 & 84.10 & 304 & 283 & 2.64 & 2.31 \\
\hline
\end{tabular}

\section{Conclusions}

In recent decades, VLC gained more attention from both commercial and academic areas. Hence, the efforts to integrate and standardize VLC to wireless infrastructures are remarkable when considering the novelties from applications (underwater, localization) to physical perspectives (LED technology, modulation). In recent scenarios, VLC-based applications have many concerns like node mobility, obstacles, shadowing, and LEDs directionality and field of view. To highlight these concerns, a novel cross layer routing protocol is proposed named MGOA for WAVLCN. In this research paper, the routes were maintained and generated on the basis of the network demands. The experimental analysis 
showed that the developed routing protocol MGOA significantly deals with obstacles and shadows, and creates alternative paths between source and destination nodes for effective data transmission. The performance metrics, such as PDR, end-to-end delay, throughput, and routing overhead, confirm that the routing protocol MGOA obtained significant performance in WAVLCN. Compared to the existing protocol, DYRP-VLC, the proposed protocol MGOA-WAVLCN almost showed 3\% to $8 \%$ improvement in PDR and reduced $0.3 \mathrm{~s}$ of end-to-end delay in data transmission. In future work, a new hybrid optimization algorithm could be developed to further improve WAVLCN performance. In addition, the proposed protocol MGOA-WAVLCN could be applied in real-world scenarios and its performance evaluated in terms of scalability, security, integrity, and confidentiality.

\begin{abstract}
Author Contributions: The paper investigation, resources, data curation, writing-original draft preparation, writing-review and editing, and visualization were done by S.V. and S.K. The paper conceptualization, software, validation, and formal analysis were done by K.R.B. Methodology, supervision, project administration, and final approval of the version to be published were conducted by A.S. and B.D.P. All authors have read and agreed to the published version of the manuscript.
\end{abstract}

Funding: This research received no external funding.

Conflicts of Interest: The authors declare no conflict of interest.

\title{
References
}

1. Cai, Y.; Guan, W.; Wu, Y.; Xie, C.; Chen, Y.; Fang, L. Indoor high precision three-dimensional positioning system based on visible light communication using particle swarm optimization. IEEE Photonics J. 2017, 9, 1-20. [CrossRef]

2. Zhang, M.; Li, F.; Guan, W.; Wu, Y.; Xie, C.; Peng, Q.; Liu, X. A three-dimensional indoor positioning technique based on visible light communication using chaotic particle swarm optimization algorithm. Optik 2018, 165, 54-73. [CrossRef]

3. Jain, S.; Mitra, R.; Bhatia, V. Adaptive precoding-based detection algorithm for massive MIMO visible light communication. IEEE Commun. Lett. 2018, 22, 1842-1845. [CrossRef]

4. Pau, G.; Collotta, M.; Maniscalco, V.; Choo, K.K.R. A fuzzy-PSO system for indoor localization based on visible light communications. Soft Comput. 2019, 23, 5547-5557. [CrossRef]

5. Jain, S.; Mitra, R.; Bhatia, V. KLMS-DFE based adaptive post-distorter for visible light communication. Opt. Commun. 2019, 451, 353-360. [CrossRef]

6. Wu, Y.; Liu, X.; Guan, W.; Chen, B.; Chen, X.; Xie, C. High-speed 3D indoor localization system based on visible light communication using differential evolution algorithm. Opt. Commun. 2018, 424, 177-189. [CrossRef]

7. Saied, O.; Ghassemlooy, Z.; Rajbhandari, S.; Burton, A. Optical single carrier-interleaved frequency division multiplexing for visible light communication systems. Optik 2019, 194, 162910. [CrossRef]

8. Chen, H.; Guan, W.; Li, S.; Wu, Y. Indoor high precision three-dimensional positioning system based on visible light communication using modified genetic algorithm. Opt. Commun. 2018, 413, 103-120. [CrossRef]

9. Zheng, H.; Chen, J.; Yu, C.; Gurusamy, M. Inverse design of LED arrangement for visible light communication systems. Opt. Commun. 2017, 382, 615-623. [CrossRef]

10. Shen, H.; Deng, Y.; Xu, W.; Zhao, C. Secrecy-oriented transmitter optimization for visible light communication systems. IEEE Photonics J. 2016, 8, 1-14. [CrossRef]

11. Wang, L.; Wang, C.; Chi, X.; Zhao, L.; Dong, X. Optimizing SNR for indoor visible light communication via selecting communicating LEDs. Opt. Commun. 2017, 387, 174-181. [CrossRef]

12. Liu, Y.; Peng, Y.; Liu, Y.; Long, K. Optimization of receiving power distribution using genetic algorithm for visible light communication. In AOPC 2015: Optical Fiber Sensors and Applications; SPIE: Bellingham, WA, USA, 2015; Volume 9679, p. 96790I.

13. Martínez-Ciro, R.A.; López-Giraldo, F.E.; Betancur-Perez, A.F.; Luna-Rivera, J.M. Design and implementation of a multi-colour visible light communication system based on a light-to-frequency receiver. Photonics 2019, 6, 42. [CrossRef]

14. Liu, H.; Zhu, P.; Chen, Y.; Huang, M. Power allocation for downlink hybrid power line and visible light communication system. IEEE Access 2020, 8, 24145-24152. [CrossRef]

15. Ulkar, M.G.; Baykas, T.; Pusane, A.E. VLCnet: Deep learning based end-to-end visible light communication system. J. Lightwave Technol. 2020, 38, 5937-5948. [CrossRef]

16. Matheus, L.M.; Vieira, A.B.; Vieira, M.A.; Vieira, L.F. DYRP-VLC: A dynamic routing protocol for wireless ad-hoc visible light communication networks. Ad Hoc Netw. 2019, 94, 101941. [CrossRef]

17. Lou, S.; Gong, C.; Wu, N.; Xu, Z. Power optimization under brightness and communication requirements for visible light communication based on MacAdam ellipse. J. Commun. Inf. Netw. 2017, 2, 28-35. [CrossRef]

18. Hong, W.X.; Wang, J.Q.; Li, W.Z. CSK hopping pattern model for visible light communication networks. Opt. Quantum Electron. 2019, 51, 1-17. [CrossRef] 
19. Sun, G.; Liu, Y.; Yang, M.; Wang, A.; Liang, S.; Zhang, Y. Coverage optimization of VLC in smart homes based on improved cuckoo search algorithm. Comput. Netw. 2017, 116, 63-78. [CrossRef]

20. Biton, C.; Arnon, S. Improved multiple access resource allocation in visible light communication systems. Opt. Commun. 2018, 424, 98-102. [CrossRef]

21. Li, F.; Wu, K.; Zou, W.; Chen, J. Analysis of energy saving ability in dimming VLC systems using LEDs with optimized SAHP. Opt. Commun. 2016, 361, 86-96. [CrossRef]

22. Ding, J.P.; Ji, Y.F. Evolutionary algorithm-based optimization of the signal-to-noise ratio for indoor visible-light communication utilizing white light-emitting diode. IET Optoelectron. 2012, 6, 307-317. [CrossRef]

23. Liu, H.; Wang, X.; Chen, Y.; Kong, D.; Xia, P. Optimization lighting layout based on gene density improved genetic algorithm for indoor visible light communications. Opt. Commun. 2017, 390, 76-81. [CrossRef]

24. Liu, H.; Lin, Z.; Xu, Y.; Chen, Y.; Pu, X. Coverage uniformity with improved genetic simulated annealing algorithm for indoor Visible Light Communications. Opt. Commun. 2019, 439, 156-163. [CrossRef]

25. Jiaan, W.; Ancheng, X.; Jintao, J.; Linyang, G. Optimization lighting layout of indoor visible light communication system based on improved artificial fish swarm algorithm. J. Opt. 2020, 22, 035701. [CrossRef]

26. Yuksel, H.; Altunay, Ö. Host-to-host TCP/IP connection over serial ports using visible light communication. Phys. Commun. 2020, 43, 101222. [CrossRef]

27. Faisal, A.; Alghamdi, R.; Dahrouj, H.; Sarieddeen, H.; Saeed, N.; Al-Naffouri, T.Y.; Alouini, M.S. Diversity Schemes in Multi-hop Visible Light Communications for 6G Networks. Procedia Comput. Sci. 2021, 182, 140-149. [CrossRef]

28. Mao, W.; Xie, H.; Tan, Z.; Liu, Z.; Liu, M. High precision indoor positioning method based on visible light communication using improved Camshift tracking algorithm. Opt. Commun. 2020, 468, 125599. [CrossRef]

29. Teng, D.; Zheng, Y.; Luo, M.; Luo, Y.; Jinyuan, W. Joint User Pairing and Subcarrier Allocation for NOMA-based Hybrid Power Line and Visible Light Communication Systems. J. Phys. Conf. Ser. 2020, 1693, 012159. [CrossRef]

30. Wu, X.; Huang, Z.; Ji, Y. Deep neural network method for channel estimation in visible light communication. Opt. Commun. 2020, 462, 125272. [CrossRef]

31. Dixit, V.; Kumar, A. Performance analysis of non-line of sight visible light communication systems. Opt. Commun. 2020, 459, 125008. [CrossRef]

32. Wu, X.; Hu, F.; Zou, P.; Lu, X.; Chi, N. The performance improvement of visible light communication systems under strong nonlinearities based on Gaussian mixture model. Microw. Opt. Technol. Lett. 2020, 62, 547-554. [CrossRef]

33. Begum, S.; Patil, N.B. An Optimal Route Discovery using Biogeography based Optimization for Vehicular Ad Hoc Networks. Int J. Comput. Netw. Commun. 2021, 13. [CrossRef]

34. Saremi, S.; Mirjalili, S.; Lewis, A. Grasshopper optimisation algorithm: Theory and application. Adv. Eng. Softw. 2017, 105, 30-47. [CrossRef]

35. Goel, N.; Grover, B.; Gupta, D.; Khanna, A.; Sharma, M. Modified Grasshopper Optimization Algorithm for detection of Autism Spectrum Disorder. Phys. Commun. 2020, 41, 101115. [CrossRef]

36. Yin, R.R.; Ghassemlooy, Z.; Zhao, N.; Yuan, H.; Raza, M.; Eso, E.; Zvanovec, S. A Multi-Hop Relay Based Routing Algorithm for Vehicular Visible Light Communication Networks. In Proceedings of the 12th International Symposium on Communication Systems, Networks and Digital Signal Processing (CSNDSP), Porto, Portugal, 20-22 July 2020; pp. 1-6.

37. Lalwani, P.; Das, S.; Banka, H.; Kumar, C. CRHS: Clustering and routing in wireless sensor networks using harmony search algorithm. Neural Comput. Appl. 2018, 30, 639-659. [CrossRef] 